\title{
Efecto de un programa de entrenamiento en casa mediado por Z0OM, en fútbol femenino durante la pandemia Covid-19, en la percepción subjetiva del esfuerzo y el apoyo a las necesidades psicológicas básicas Effect of an Z0OM mediated home training program in women's soccer during the Covid-19 pandemic on the Rating of Perceived Exertion and support for basic psychological needs \\ Ivo Jiménez-Barreto, Pablo José Borges Universidad de La Laguna (España)
}

\begin{abstract}
Resumen. El entrenamiento individualizado no presencial resultahabitual en deportesindividuales y colectivos, especial mente en aquellos más dependientes de un soporte condicional. En este trabajo se presenta un programa de entrenamiento virtual grupal (PEVG) en casa, mediado por la aplicación Zoom, y desarrollado por un equipo de fútbol femenino durante el confinamiento por la pandemia Covid-19. El propósito de este estudio ha sido valorar el impacto del PEVG en el estímulo condicional, estado de ánimo, y el apoyo psicosocial. Para valorar su efecto en los dos primeros aspectos, se cumplimentó un cuestionario wellness al terminar cada sesión. Al finalizar el confinamiento se aplicó una escala de medición de apoyo a las necesidades psicológicas básicas (NPB). Los resultados procesuales del cuestionario wellness muestran valores medios y altos, y estabilidad en todos sus ítems, siendo el estado de ánimo el que obtiene valores superiores y menor fluctuación. Asimismo, se aprecian diferencias en la percepción de carga atendiendo a los rasgos del perfil de las jugadoras, puesto específico (defensas-atacantes), y edad (jóvenes-senior). Al finalizar la experiencia, la valoración del estímulo condicional permite inferir que la percepción de esfuerzo parece haber estado influenciada por las limitaciones de movilidad impuestas y la duración del estado de confinamiento, y los tipos de sesiones empleados. Respecto al apoyo a las N PB, se constata una alta puntuación en la dimensión apoyo a la relación social, lo que teniendo en cuentala situación de separación de las jugadoras se relaciona con el empleo de la aplicación telemática.
\end{abstract}

Palabras clave: entrenamiento virtual, motivación, necesidades psicológicas básicas, preparación física, fútbol femenino.

\begin{abstract}
Individualized non-face-to-face training is common in individual and group sports, especially in those more dependent on conditional support. This paper presents a virtual group training program (PEVG) at home, mediated by Zoom application, and developed by a women's soccer team during the Covid- 19 confinement. The purpose of this study has been to assess the impact of PEVG on conditional stimulation, mood, and psychosocial support. To assess its effect on the first two aspects, a wellness questionnaire was completed at the end of each session. At the end of the confinement period, support for basic psychological needs scale (NPB) was applied. The procedural results of the wellness questionnaire show medium and high values, and stability in all its items, being the mood the onethat obtains higher values and lessfluctuation. Likewise, there are differences in the Rating of Perceived Exertion according to the characteristics of the players' profile, specific position (defenders-attackers), and age (young-seniors). At the end of the experience, the assessment of the conditional stimulus allows us to infer that the perception of effort seems to have been influenced by the mobility limitations imposed and the duration of the confinement state, and the types of sessions used. Regarding support for NPBs, a high score is found in the dimension support for the social relationship, which, taking into account the situation of separation of the players, is related to the use of the telematics application.
\end{abstract}

Keywords: Virtual training, motivation, Basic Psychological N eeds, fitness training, women's football

\section{Introducción}

El entrenamiento no presencial donde se aporta al deportista las sesiones a realizar, y éste las realiza de manera individual y autónoma, es una práctica habitual

Fecha recepción: 30-07-20. Fecha de aceptación: 19-01-21

Pablo José Borges

pborgesh@ull.edu.es tanto en los deportes individual es como de equipo, aunque en éstos últimos se suele emplear en periodos vacacionales y es desarrollado de manera asincrónica e individual. Lo que no es habitual es que las jugadoras de un equipo de fútbol puedan realizarlo de manera individual y simultánea, de ahí el interés de este estudio que explora los efectos de un programa de entrenamiento virtual grupal (PEVG). Su aplicación durante el período de confinamiento por el Covid-19, ha sido posible por el empleo de la plataforma Zoom ${ }^{\mathrm{TM}}$ (Zoom Video 
Communications, San Jose, CA), caracterizada por la pantalla partida que permitía la interacción visual y auditiva entre las jugadoras, y entre estas y el cuerpo técnico.

La motivación, entendida como una serie de procesos que dan cuenta de la intensidad, dirección y persistencia del esfuerzo de un individuo por conseguir una meta (Robbins, 2004), es una característica psicológica multidimensional, que se ve influenciada tanto por los aspectos internos de la persona (preferencias, deseos, temores, etc. ), como por las vivencias externas de su entorno (aceptación social, amistades, habilidades, etc.). En la planificación de programas de actividad física (AF) es una variable importante a tener en cuenta porque incidirá en la forma y modo de participación del individuo en ese programa y en su rendimiento, en tanto que tiene influencia en aspectos como la iniciación, dirección, magnitud, perseverancia, continuidad, y calidad de la conducta dirigida a una meta (M aehr $\&$ Zusho, 2009). Esta cuestión aconseja contemplar estrategias motivacionales en los procesos de planificación y entrenamiento deportivo que favorezcan la adherencia a la práctica deportiva y la mejora del rendimiento.

El carácter dinámico de la motivación la hace sensible a un gran número de variables, relacionadas algunas de ellas con las circunstancias que envuelven una determinada actividad. Desde la teoría de la autodetermina ción (TAD) (Ryan \& Deci, 2019), y más concretamente desde la mini-teoría de las N ecesidades Psicológicas Básicas (NPB), se postula que el comportamiento humano es motivado por tres necesidades primarias y univer sales: autonomía (actuar con libertad adquiriendo un papel protagonista), competencia (sentimiento de eficaz y capacidad para afrontar las tareas), y la rela ción con los demás (sentirse conectado e integrado con otras personas). La satisfacción de estas necesidades es esencial para el crecimiento, la integración, el desarroIlo social y el bienestar personal, y genera un aumento en la motivación (Deci \& Ryan 2000; Ryan $\&$ Deci 2000). En esta línea de investigación, el esfuerzo es entendido como una forma de «compromiso» 0 «determinación», y éste ha sido relacionado con la motivación y la satisfacción de las N PB en el contexto del ejercicio físico (Duda \& H all, 2001; Tenenbaum et al., 2001), influyendo además en predicción de la percepción del esfuerzo (M oreno-M urcia et al., 2012), la diversión y el aburrimiento (Bæena-Extremera et al., 2016).

El modelo de laTAD se ha convertido en un referente muy utilizado en los estudios que examinan la relación entre motivación y AF; sin embargo, ninguno de los trabajos analizados en la revisión sistemática sobre este modelo (Salazar-Ayala \& Gastélum-Cuadras, 2020; Sevil et al., 2016), se enfocó de manera específica sobre la necesidad de relaciones sociales. El apoyo a las NPB se asocia al aumento de la motivación intrínseca (Cecchini, Carriedo, \& M éndez-Giménez, 2019) y a la intención de participar en AF (Sevil et al., 2014), lo que invita a tener en cuenta esta variable motivacional en el diseño de los entrenamientos virtuales. Estrategias de inter vención como el diseño de actividades y el discurso verbal son considerados relevantes para conseguir el apoyo a las N PB (Sevil et al. , 2016), a los que se puede añadir las opciones metodológicas y la configuración de los entornos de práctica que promuevan ese apoyo. Dentro de esos entornos Balaguer (2010) y González et al., (2015) destacan la importancia del apoyo ambiental social para que se produzca el desarrollo óptimo de la persona.

El uso de la pantalla partida en esta experiencia se justifica por la posibilidad que ofrece este tipo de herra mienta digital para integrar en un mismo plano, múltiples conductas motrices sincronizadas o no. Esta mediación tecnológica, ha generado un modelo de PEVG con un espacio y tiempo específico de interacción, que trasciende el aislamiento establecido en el periodo de confinamiento. Junto con las implicaciones psicológicas respecto a la motivación, la continuidad del entrenamiento a través de la virtualidad ha permitido asegurar los beneficios adicionales de las rutinas vitales, y la realización de AF diaria siguiendo las directrices de la OMS (2010); y para mitigar el riesgo de empeoramiento físico (Blauwet et al., 2020).

En este escenario de interacción virtual la presencia en la pantalla también del entrenador, le confiere a éste, un refuerzo como líder del grupo y presumiblemente aumentará la intensidad del trabajo desarrollado por las deportistas como ha sido acreditado en contexto de entrenamiento presencial por Falces, Casamichana y Suarez-Arrones(2013). También los estudios deA guirre, Tristán, López, Tomás, y Zamarripa (2016), Balaguer et al. (2012), y Cantú-Berrueto et al. (2016), han evidenciado en contextos presenciales en fútbol que el empleo de un estilo interper sonal por parte del entrenador promueve el apoyo a las NPB. En un contexto virtual, el ofrecimiento voluntario de las propias imágenes genera un espacio de ejercitación compartida, que refuerza el sentimiento de grupo, lo que unido a la presencia del entrenador y el empleo de un estilo interpersonal puede generar un apoyo efectivo a las N PB y, por tanto, a la motivación de las jugadoras. 
En relación al estímulo condicional del entrenamiento, Viru y Viru (2003) afirman que es difícil relacionar el entrenamiento con las mejoras o déficits en el rendimiento de competición sin un control estricto de las variables de la carga de entrenamiento. Por tanto, es necesario atender a la cuantificación de esta carga desde su doble vertiente (carga externa), conjunto de actividades que constituyen launidad de entrenamiento y (carga interna) el grado de exigencia que representa para el organismo (Pérez, 2017). Por ello, es relevante cuantificar individualmentelacargainternade cadatarea, desde diferentes ópticas y metodologías. Entre ellas, se encuentra la Percepción Subjetiva del Esfuerzo (RPE del inglés Rating of Perceived Exertion), definida como la intensidad subjetiva de esfuerzo, tensión, malestar y/ 0 fatiga que se experimenta durante el ejercicio físico influenciado por factores psicológicos, emocionales y contextuales (Pescatello et al., 2014). Se trata de un método válido, económico y fácil de aplicar (LosArcos et al., 2014), producto de la investigación de Borg y Dahlstron (1962) al alcance de cualquier equipo independientemente del nivel competitivo y sus posibilidades económicas (M uñoz, Castillo, \&Yanci, 2018).

La RPE aporta no solo información genérica del esfuerzo percibido, sino también relaciona dicho esfuerzo con otras variables como la Frecuencia Cardíaca o el Vo2M ax (Alexiou \& Coutt, 2008), y laconcentración de lactato en sangre (Borg, 1998; Hetzler et al., 1991). Asimismo, ha sido correlacionada con la eval uación externa de la carga en deportes colectivos e intermitentes como el rugby (Lovell et al., 2013), el fútbol australiano (Gallo et al., 2015) y el fútbol (Castellano, Echeazarra, \& Estéfano, 2017), mediantetecnologíaGPS y RPE.Y específicamente durante trabajos intermitentes usando juegos reducidos (Gaudino et al. 2015); 0 al comprobar la asociación entre RPE y distancia total cubierta en jugadores de fútbol semiprofesionales (Casamichana \& Castellano, 2013). Esto ha llevado a Melim et al. (2020) a recomendar su empleo para el seguimiento del entrenamiento en casa ante las limita ciones generadas por el COVID-19.

La RPE se ha convertido en una escala y temática representativa de la literatura científica actual sobre el seguimiento del entrenamiento deportivo, para la comparación de diferentes situaciones en deportes individuales y colectivos (Alexiou \& Coutts, 2008; Borresen \& Lambert, 2008; Coutts et al, 2009). También para la cuantificación de lacargainternadeentrenamiento (falta 0 exceso) y su relación con la pérdida de rendimiento físico (Rebelo et al. , 2016) en sesiones de entrenamien- to (Álvarez et al., 2009); y la discriminación entre diferentes tipos de entrenamiento y la situación de competición de fútbol sala (Pascual et al., 2016). Todo ello, sin olvidar que el fútbol es un deporte de equipo en el que se aprecian diferencias individuales entre los jugadores que se reflejan en la función que cada uno realiza en el campo (Del Pino, Gómez, \& Moreno, 2015). Además la RPE permite monitorear a cada jugador y aportar datos inmediatos para comparar las respuestas de estos con la planificación (Álvarez \& M urillo, 2016), debido a la dificultad de controlar la carga interna en entrena mientos de metodología específica o integrada en deportes colectivos (Pérez, 2017). Este tipo de valoración individualizada ayuda a los entrenadores a controlar el proceso de entrenamiento y mejorar el rendimiento (Alexiou \& Coutt, 2008). Lo que se traduce en la publicación de al rededor de 200 artículos al año y su empleo en aproximadamente más de un millón de participantes.

Por tanto, los objetivos de este estudio fueron: 1) valorar la evolución de la percepción de carga física y mental inducida por los diversos tipos de sesiones, y en función de las tres fasesen las que se estructuró el PEVG, considerando la experiencia, edad y posición de las jugadoras; 2) analizar la percepción final de las jugadoras acerca del apoyo a las NPB (relación social, competencia y autonomía), y de la carga física y mental al finalizar el PEVG.

\section{M etodología}

La investigación ha seguido un diseño descriptivo y correlacional de carácter transversal, constituyéndose como un estudio instrumental siguiendo un diseño ex post facto retrospectivo (M ontero \& León, 2007).

\section{Participantes}

Para la realización de este estudio participaron 19 deportistas pertenecientes a un equipo femenino de un club de fútbol de la isla deTenerife, que fueron seleccionadas por criterios de conveniencia, disponibilidad participativa y recursos propios, con edades comprendidas entre 13 y 34 años ( $M=17,63$ y $D T=4,45$ años) y con experienciadeportivaen fútbol $(M=9,31$ y $D T=4,57$ años). También participó el preparador físico responsa ble de los entrenamientos, con una experiencia profesional de más de 10 años. Asimismo, estaban presentes en pantalla el resto del cuerpo técnico. Antes de la investigación se informó del procedimiento y se solicitó su consentimiento a las deportistas, siguiendo las pautas marcadas en la Declaración de Helsinki (Asociación 
Médica M undial, 2017). En laTabla 1 se muestra el perfil deportivo de las jugadoras de esta experiencia.

\begin{tabular}{|c|c|c|c|c|}
\hline Jugadora & Edad & Experiencia & Categoría & Puesto Específico \\
\hline J1 & 18 & 9 & Senior & Portera \\
\hline 12 & 19 & 13 & Senior & M ediocentro Defensivo \\
\hline j3 & 14 & 9 & Cadete & Media punta \\
\hline j4 & 18 & 11 & Senior & Media punta \\
\hline$j 5$ & 16 & 4 & Juvenil & Medio Centro Defensivo \\
\hline 16 & 14 & 9 & Cadete & Defensa Central \\
\hline 17 & 18 & 7 & Senior & Extremo \\
\hline 18 & 16 & 1 & Juvenil & Extremo \\
\hline 19 & 15 & 3 & Cadete & Extremo \\
\hline j10 & 15 & 4 & Cadete & Extremo \\
\hline j11 & 20 & 16 & Senior & Portera \\
\hline j12 & 13 & 4 & Infantil & Delantera \\
\hline 13 & 16 & 10 & Juvenil & Mediocentro 0 fensiva \\
\hline 14 & 18 & 10 & Senior & Defensa Central \\
\hline 15 & 19 & 10 & Senior & Lateral \\
\hline J16 & 18 & 9 & Senior & Lateral \\
\hline j17 & 19 & 15 & Senior & Lateral \\
\hline j18 & 15 & 4 & Cadete & Lateral \\
\hline j19 & 34 & 17 & Senior & Delantera \\
\hline
\end{tabular}

\section{Técnicas de recogida de datos}

Para el seguimiento del proceso de entrenamiento las deportistas cumplimentaron al final de cada sesión y del periodo de confinamiento un cuestionario wellness generado ad hoc, que es el que se venía utilizando de manera habitual el club sobre el estado de ánimo, percepción de la carga del entrenamiento, y nivel de esfuerzo. El cuestionario wellness estaba compuesto por tres ítems con un formato de respuesta tipo likert de cinco niveles. Los enunciados de los ítems fueron los siguientes: 1. ¿Cómo te has encontrado en general durante el entrenamiento? 2. ¿Cuánto te ha representado la carga del entrenamiento realizado? 3. ¿Cuál ha sido tu nivel de esfuerzo durante el entrenamiento?

Asimismo, se aplicó al final del proceso de entrena miento la escala de medición de apoyo a las necesidades psicológicas básicas de Vlachopoulos \& Michailidou (2006), con la intención de conocer el grado de motiva ción generado en las jugadoras. La escala consta de 12 ítems con un formato de respuesta en 5 niveles Tipo Likert, siendo el nivel 1 «Totalmente en desacuerdo» y el nivel 5 «Totalmente de acuerdo». Los ítems establecen dimensiones en base a la'autonomía' ( $1,4,7$ y 10$)$, la'competencia' $(2,5,8$ y 11$)$ y' relación con los demás' $(3,6,9$ y 12). Según el procedimiento aplicado de consistencia internaA Ipha de Cronbach, la escala empleada muestra un índice de fiabilidad aceptable de 0,837 (McMillan, 2008). Ambos cuestionariosfueron auto-administrados y cumplimentados mediante aplicación online, y la duración de respuesta aproximada delatoma de datos fue inferior a 5 minutos.

\section{Procedimiento}

Antes del confinamiento, el equipo venía realizando un microciclo competitivo semanal de tres sesiones de entrenamiento, iniciándose dos de ellas con un trabajo de fuerza coadyudante (Gómez et al., 2018), además del partido el fin de semana. Una vez efectivo el estado de alarma (R.D, 463/ 2020 de 14 de marzo de 2020), y con la implantación del periodo de confinamiento, se inició el PEVG a partir del 23 de marzo hasta el 10 de mayo (35 sesiones), siguiendo una frecuencia de seis sesiones semanales (Figura 1 ).

\begin{tabular}{|c|c|c|c|c|c|c|}
\hline Lunes & Martes & Miércoles & Jueves & Viernes & Sábado & Domingo \\
\hline Sesión tipo 1 & \multicolumn{2}{|c|}{ Sesión tipo 2} & & Sesión tipo 3 & Sesión tipo 2 & \multirow{6}{*}{$\mathrm{RCO}$} \\
\hline \multicolumn{3}{|c|}{ ANM } & & ANM & ASM & \\
\hline $\begin{array}{c}\text { Bloque } 1 \\
\text { FFO }\end{array}$ & \multicolumn{2}{|c|}{$\begin{array}{c}\text { Bloque } 1 \\
\text { FFC }\end{array}$} & & $\begin{array}{c}\text { Bloque } 1 \\
\text { FMD }\end{array}$ & Bloque $3 \mathrm{CCCl}$ & \\
\hline $\begin{array}{c}\text { Bloque } 2 \\
\text { FRC }\end{array}$ & $\begin{array}{c}\text { Bloque } 2 \\
\text { HIIT }\end{array}$ & $\begin{array}{l}\text { Bloque } 2 \\
\text { EMOM }\end{array}$ & & $\begin{array}{c}\text { Bloque } 2 \\
\text { CTM }\end{array}$ & Bloque 2 ESM & \\
\hline $\begin{array}{c}\text { Bloque } 3 \\
\text { CEA }\end{array}$ & \multicolumn{2}{|c|}{$\begin{array}{c}\text { Bloque } 3 \\
\text { FCP }\end{array}$} & & $\begin{array}{c}\text { Bloque } 3 \\
\text { FCP }\end{array}$ & \multirow{2}{*}{$\begin{array}{c}\text { Bloque } \\
\text { Complementario } \\
\text { EAN y LMF }\end{array}$} & \\
\hline $\begin{array}{c}\text { Bloque } \\
\text { complementario } \\
\text { LMF }\end{array}$ & \multicolumn{2}{|c|}{$\begin{array}{c}\text { Bloque } \\
\text { complementario } \\
\text { EAN }\end{array}$} & & $\begin{array}{c}\text { Bloque } \\
\text { complementario } \\
\text { EAN }\end{array}$ & & \\
\hline
\end{tabular}

Figura 1. Desarrollo estructura semanal del microciclo atendiendo a la distribución de los bloques de trabajo. Leyenda: activación neuromuscular (ANM); activación soporte musical (ASM); contenidos neuromusculares optimizadores (FFO); fuerza funcional con autocargas (ASM); contenidos neuromusculares optimizadores (FFO); fuerza funcional con autocargas
(FFC); fuerza orientada al movimiento deportivo (FMD); fuerza resistencia mediante circuito (FRC), High-intensity interval training (HIIT), Every Minute on the Minute (EMOM), circuito de tareas multiarticulares (CTM); resistencia mediante coreografías con soporte musical (ESM); fuerza compensatoria (FCP), ejercicios de core, cadera e isquiosurales (CCCI), ejercicios esl abones débiles 0 antagonistas (CEA); estiramientos (EAN); liberación miofascial (LMF).

Para la configuración del PEVG se consideraron tres sesiones tipo distribuidas durante la semana (Figura 1), siendo caracterizada la sesión Tipo 1 por incidir preferentemente sobre los niveles neuromusculares de fuerza; la sesión Tipo 2 por mantener prioritariamente los niveles cardiovasculares de resistencia mediante diferentes métodos; y la sesión Tipo 3 por dar prioridad a movimientos relacionados con el gesto deportivo específico, y optimizar los rangos de amplitud articular. En cada tipo de sesión se sigue una estructura base de contenidos organizados en diferentes bloques directamente relacionados con las demandas del juego:

-Bloque inicial: activación neuromuscular (ANM) y activación con soporte musical (ASM);

-Bloque 1 en el que se discriminaron contenidos de fuerza: fuerza funcional (Boyle, 2017) con sobrecarga enfocadaalos contenidosneuromuscularesoptimizadores (FFO), fuerza funcional con autocargas (FFC), fuerza orientada al movimiento deportivo (FMD) mediante habilidades básicas de movimiento (M oras, 2017a);

-Bloque 2 donde se discriminaron contenidos de resistencia: fuerza resistencia mediante circuito (FRC), tareas metabólicas a altas intensidades en forma de HighIntensity Interval Training (HIIT), Every Minute on the Minute (EMOM), circuito de tareas multiarticulares (CTM) para mejorar los rangos de amplitud articular (M oras, 2017b), resistencia mediante coreografías con 
soporte musical (ESM);

-Bloque 3 en el que se discriminaron los siguientes contenidos: Tareas compensatorias neuromusculares: fuerza compensatoria (FCP), ejercicios específicos de core, cadera e isquiosurales (CCCI), haciendo hincapié en los eslabones débiles 0 antagonistas (CEA); y tareas complementarias: estiramientos (EAN) siguiendo el protocolo de Anderson (2017) o liberación miofascial (LMF).

Para apoyar la cohesión del grupo, los domingos se propusieron diferentes retos cooperativos (RCO), en los que se requería la participación de algún familiar para su superación. Las temáticas de los retos fueron variadas: condición física, habilidad técnica o nutrición, con la intención de apoyar la N PB de autonomía. Asimismo, una 0 varias jugadoras se encargaban de presentar y de dirigir las tareas siguiendo un estilo de microenseñanza (Siedentop, 1998).

Las sesiones virtuales se desarrollaron a través de la plataformaZoom $^{\mathrm{TM}}$ (ZoomVideo Communications, San Jose, CA) conectándose las 19 jugadoras y el cuerpo técnico, eligiendo las jugadoras el espacio más adecuado de sus domicilios para la práctica deportiva (68\% salón y 31\% azotea). El preparador físico enviaba previamente la sesión a las jugadoras; al conectarse les preguntaba sobre su estado físico, mental y emocional. En función de las respuestas, se aportaban pautas individuales de ajuste de la intensidad de trabajo y ejercicios alternativos a aquella jugadora que hubiera señal ado algún tipo de limitación. Y por último, se resolvían las posibles dudas respecto los ejercicios propuestos antes de iniciar la sesión. Posteriormente se procedía a realizar un ca lentamiento estándar de 5-10 minutos, una parte principal de 40-45 minutos en la que el cuerpo técnico marcaba el tiempo de trabajo, ofrecía feedback y alentaba la práctica. Las jugadoras, durante la sesión, podían beber líquidos «at libitum» al término de cada bloque de actividades. Parafinalizar se procedíaa desarrollar unavuelta a la calma estándar, compuesta de trabajo de core y estiramientos siguiendo el método deAnderson (2017), aprovechando este lapso de tiempo para solicitar la cumplimentación del cuestionario wellness.

\section{Análisis de datos}

Inicialmente, y tomando como referencia a todo el grupo se procedió con estadística descriptiva (media y desviación típica) para valorar la evolución de las puntuaciones medias en los ítems del cuestionario wellness, también se exploró la percepción de las jugadoras atendiendo a los tipos de sesiones (Figura 1), y a las tres fases en las que se estructuró el PEVG : Fase Inicial (que comprende las 8 sesiones en las que se recopilaron da tos entre el 23 de marzo y el 11 de abril), Fase M edia (las 10 sesiones en las que se recopilaron datos entre el 13 y el 25 de abril), y Fase Final (las 9 sesiones en las que se recopilaron datos entre el 27 de abril y el 9 de mayo).

Con la intención de indagar la posible incidencia de los rasgos del perfil de las jugadoras, en función de las fases del programa y el tipo de sesión, se consideraron como variables dependientes los siguientes parámetros normativos: experiencia (considerando novatas, aquellas deportistas con menos de 9 años de experiencia y expertas aquellas con más de esa cifra), puesto específico de lasjugadoras, organizadasen dosgrupos, defensoras (porteras, centrales, laterales y mediocentros defensivos) y atacantes (mediocentro ofensivos, extremos, mediapuntas y delanteras), y edad considerando jóvenes, aquellas deportistas menores de 17 años y senior el resto, siguiendo un criterio estadístico no teórico). Para determinar el tipo de análisis a realizar, se aplicó la prueba de normalidad de Shapiro-W ilk, encontrando que al gunos valores eran no normales por lo que se procedió con estadística no paramétrica y la prueba $U$ de Mann W hitney para muestras relacionadas cuando se toma como referencia el grupo completo, y no relacionadas cuando se atiende al perfil de las jugadoras.

En cuanto a la valoración post intervención, se ana lizaron las puntuaciones finales promedio del cuestiona rio wellnessy la escalaN PB, considerando el grupo completo y, por otra parte, atendiendo a los parámetros de los perfiles seleccionados. Para todos los análisis se empleó el paquete estadístico R 4.0.2 (2017), estableciendo como nivel de significación $p<0,05$.

\section{Resultados}

La Figura 2 muestra la evolución de los ítems del cuestionario wellness (escala 1-5), tomando como referencia las puntuaciones medias del equipo en su conjunto y la fluctuación individual de cada jugadora (DT) en cada sesión del PEVG. Se observa una mayor estabilidad y puntuación en el ítem estado de ánimo, así como

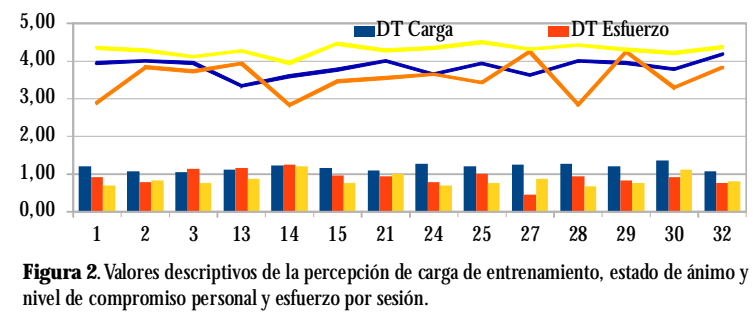


mayor dispersión a lo largo del proceso en el ítem esfuerzo, mientras que para las puntuaciones individuales de cada sesión se aprecia mayor variabilidad en el ítem carga.

Considerando los tipos de sesiones del PEVG, y tomando como referencia todo el grupo, no se aprecian diferencias significativas. En cuanto a las fases en las que se estructuró el PEVG, y tomando como referencia todo el grupo, se encuentran diferencias significativas $(p<0,001)$ entre la percepción de esfuerzo entre la fase inicial y media, a favor de la fase media $\left(M_{E 1}=3,44<\right.$ $\left.M_{E 2}=3,55\right)$, y entre la fase media y final a favor de esta última $\left(M_{E 2}=3,55<M_{E 3}=3,74\right)$; mientras que en el estado de ánimo general, se aprecia significación similar entre la fase inicial y media, a favor de la segunda $\left(M_{G 1}=4,22<M_{G 2}=4,38\right)$, y entre la fase media y final a favor de la primera $\left(M_{G 2}=4,38>M_{G 3}=4,31\right)$.

Al segmentar la muestra considerando los parámetros normativos, se hallan diferencias significa tivas en el ítem carga durante las sesiones de la Fase 1 en función del puesto específico ( 3,51 vs. $4.59 ; p<0,05)$, puntuando más alto las atacantes (Tabla 2).

Por otra parte, y a partir de los resultados de la Tabla 2, se realizó un análisis complementario de comparación de medias para muestras relacionadas entre las diferentes fases del programa. Se aprecian diferencias significativas en el caso de los parámetros normativos edad y puesto específico en las puntuaciones de los ítems esfuerzo y estado de ánimo. En el caso de las jóvenes, se aprecian diferencias entre las puntuaciones del ítem esfuerzo a favor de la fase 2 respecto a la 1, y de la fase 3 respecto a la $2(p<0,05)$. En el caso de las expertas, se aprecian diferencias entre las puntuaciones del ítem esfuerzo a favor de la fase 2 respecto a la 1 y de la fase 3 respecto a la $2(p<0,05)$, mientras que en el caso del ítem estado de ánimo, se aprecian diferencias a fa vor de la fase 2 respecto a la 1 y de la fase 2 respecto a la $3(p<0,05)$.

\begin{tabular}{|c|c|c|c|c|c|c|c|c|c|}
\hline & & Edad & & & Experiencia & & Pues & to específic & \\
\hline & Joven & senior & d & Novato & Experto & d & Defensa & Ataque & $d$ \\
\hline $\mathrm{Pr} r_{-} \mathrm{Cl}$ & 4 & 3,58 & 0,32 & 3,97 & 3,49 & 0,23 & $3,51^{*}$ & $4,09 *$ & $-0,53$ \\
\hline $\mathrm{Pr}^{-} \mathrm{E} 1$ & 3,35 & 3,52 & $-0,07$ & 3,58 & 3,23 & 0,42 & 3,53 & 3,33 & 0,18 \\
\hline $\mathrm{Pr}_{-}^{-} \mathrm{Gl}$ & 4,24 & 4,21 & 0,11 & 4,32 & 4,08 & 0,13 & 4,21 & 4,24 & $-0,04$ \\
\hline $\mathrm{Pr}_{-} \mathrm{CC} 2$ & 4,17 & 3,58 & 0,71 & 3,74 & 3,95 & 0,42 & 3,52 & 4,21 & $-0,61$ \\
\hline $\mathrm{Pr}_{-}^{-} \mathrm{E2}$ & $3,56^{\wedge}$ & $3,54^{\wedge}$ & 0,22 & $3,65^{\wedge}$ & $3,42^{\wedge}$ & 0,53 & $3,43^{\wedge}$ & $3,68^{\wedge}$ & $-0,28$ \\
\hline $\mathrm{Pr}_{-}^{-} \mathrm{G} 2$ & $4,33^{\wedge}$ & $4,42^{\wedge}$ & 0,30 & 4,35 & $4,42^{\wedge}$ & 0,23 & $4,30^{\wedge}$ & $4,47^{\wedge}$ & $-0,21$ \\
\hline $\mathrm{Pr}_{-} \mathrm{C} 3$ & 4,27 & 3,6 & 0,48 & 3,93 & 3,83 & 0,26 & 3,32 & 4,42 & $-0,99$ \\
\hline $\mathrm{Pr}_{-}^{-} \mathrm{E} 3$ & $3,73^{\wedge}$ & $3,75^{\wedge}$ & 0,03 & $3,90^{\wedge}$ & $3,53^{\wedge \wedge}$ & 0,43 & $3,71^{\wedge}$ & $3,78^{\wedge}$ & $-0,08$ \\
\hline $\mathrm{Pr}_{-} \mathrm{G}_{3}$ & $4,33^{\wedge}$ & $4,30^{\wedge}$ & 0,09 & 4,43 & $4,17^{\wedge}$ & 0,20 & $4,24^{\wedge}$ & $4,39^{\wedge}$ & $-0,18$ \\
\hline
\end{tabular}

Considerando los tres tipos de sesiones (ver Figura 1), se aprecian diferencias significativas en el factor pues- to específico en el ítem carga (Tabla 3), mostrando las atacantes valoressuperiores para las sesiones tipo 2 (4,24 vs. 3,38; $p<0,001)$, y tipo 3 (4,12 vs. 3,35; $p<0,05)$. A partir de los resultados de laTabla 3 , se realizó una comparación de medias para muestras relacionadas entre los diferentes tipos de sesiones, tomando como referencia las variables normativas (edad, experiencia y puesto específico). Únicamente se aprecian diferencias significativas en las puntuaciones del ítem esfuerzo, en el caso de las deportistas novatas, a favor de las sesiones tipo 1 respecto a las tipo $2(p<0,05)$.



Las puntuaciones promedio finales de la medición de las N PB (Vlachopoulos\& M ichailidou, 2006), tomando como referencia a todo el grupo (Figura 3), muestran la siguiente secuencia de puntuación: relaciones sociales $(M=4,78, D T=0,41)$, competencia $(M=4,62$, $\mathrm{DT}=0,39)$, y autonomía (DT=4,29, DT=0,67). Respecto a los resultados finales del cuestionario wellness (Figura 3), la secuencia es la siguiente: el ítem esfuerzo $(M=4,42 D T=0,61)$, estado de ánimo $(M=4,11$ $\mathrm{DT}=0,88)$, y carga percibida $(M=3,90, \mathrm{DT}=0,57)$.

Figura 3. Valores promedios post intervención de las dimensiones de la escala N PB y los ítems del cuestionario wellness considerando todo el equipo $(n=19)$.

Al analizar los resultados finales, segmentando el grupo por los parámetros normativos, se aprecian diferencias significativas en el factor puesto específico y edad en el ítem carga (Tabla 4), siendo las percepciones superiores en las atacantes ( 4,23 vs. 3,$45 ; p<0,01)$, y en las jóvenes ( 4,12 vs. 3,$59 ; p<0,001)$. Además se observa cómo las puntuaciones más bajas se relacionan con el ítem esfuerzo. 
Tabla 4.

Valores descriptivos finales y contraste de medias de la percepción post intervención de los ítems del

cuestionario well Iness en función de la edad, experiencia y puesto específico. Edad Experiencia Puesto específico Joven senior $d$ Novato Experto $d$ Defensa Ataque $d$ $\begin{array}{cccccccccc}\text { Carga } & 4,12 * & 3,59 * & 0,44 & 3,91 & 3,70 & 0,19 & 3,45^{* *} & 4,23 * * & -0,70 \\ \text { Esfuerzo } & 3,51 & 3,60 & 0,03 & 3,70 & 3,38 & 0,07 & 3,56 & 3,56 & 0,01\end{array}$

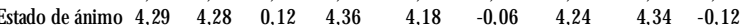
${ }^{*} p<0,01 ; * * p<0,001 ; d: V a l o r e s$ de la prueba $d$ de Cohen.

\section{Discusión}

El análisis de la evolución de las puntuaciones medias del cuestionario wellness (Figura2), tomando como referencia al grupo en su conjunto, muestra una ausencia de diferencias significativas a lo largo de cada una de las sesiones en los ítems del cuestionario wellness. Ello permite pensar, que la respuesta de las jugadoras ha sido homogénea y las ligeras fluctuaciones de las RPE durante el PEVG, pueden deberse a factores individuales como la edad, el puesto específico y la experiencia, relacionadas con la situación personal y/ o las características de personalidad de cada deportista. En cual quier caso, el hecho de que el estado de ánimo se haya mantenido estable y con una mayor puntuación, puede estar relacionado con la dinámica de desarrollo grupal de las sesiones, mediada por la plataforma Zoom, que permitió mantener el contacto entre las jugadoras y la cohesión del grupo. Así como ofrecer a las deportistas un espacio de disrupción de la rutina del confinamiento. Por otra parte, la dispersión que muestra a lo largo del proceso el ítem esfuerzo, se vincula a las demandas diferenciadas que provocan los diferentes tipos de sesiones diseñados. En cuanto a las puntuaciones individua les, la mayor variabilidad en el ítem carga se relaciona con el alto grado de heterogeneidad del grupo en función de sus características individuales (edad, experiencia y puesto específico).

En cuanto a las fases de entrenamiento y tomando como referencia al grupo, se observan diferencias significativas $(p<0,001)$ que indican un aumento secuencial de la percepción de esfuerzo de la fase media respecto a la inicial, y de la final respecto a la media. Esto se puede relacionar con el efecto acumulativo de las limitaciones impuestas por el estado de confinamiento domiciliario. Lo que puede traducirse en una disminución del estado de forma de las deportistas provocado por las restricciones que impone el domicilio habitual para la práctica de una actividad física variada, tal y como encuentran con atletas Seguí-U rbaneja et al. (2021), o la no disposición en casa de equipamiento específico y acorde con el entrenamiento deportivo, así como el triple rol femenino aludido por M oscoso (2019). Respecto al estado de ánimo, se observa un aumento del mismo en la fase media respecto a la inicial, y de la media respecto a la final, lo que puede estar señalando que las percepciones de la fase inicial pudieron estar afectadas por la gran incertidumbre que se generó al inicio del confinamiento. Estabilizándose la percepción en la fase media, una vez que las deportistas se fueron adaptando a los cambios en la rutina diaria del estado de confinamiento (Seguí-U rbaneja et al. 2021), y disminuyendo en la fase final por la prolongación de un modelo de vidaque constreñía la convivencia al domicilio y el contexto familiar en un espacio reducido. Por último, y respecto a las puntuaciones medias de todo el grupo, no se han encontrado diferencias significativas en los ítems del cuestionario wellness entre los diferentes tipos de sesiones. Este aspecto puede estar vinculado al alto grado de heterogeneidad del perfil de las jugadoras.

Basándonos en esta heterogeneidad nos llevó a proseguir con un análisis segmentando la muestra por parámetros normativos relacionados con el perfil de las jugadoras (edad, experiencia y puesto específico). En cuanto a las fases del PEVG y considerando el puesto específico (Tabla 2), se hallan diferencias significativas en el ítem carga durante las sesiones de la fase $1(3,51$ vs. 4,59; $p<0,05$ ), siendo las atacantes las que perciben una mayor carga. En cuanto al tipo de sesiones (Tabla3), tanto en las sesiones tipo 2 de trabajo prioritario de la resistenciacardiovascular $(4,24$ vs. 3,38; $p<0,001)$, como las sesiones tipo 3 que priorizan los movimientos específicos del gesto deportivo (4,12 vs. 3,35; $p<0,001)$, son de nuevo las atacantes las que perciben un mayor nivel de carga. Creemos que estos datos pueden estar relacionados con que el subgrupo de jugadoras atacantes que venía acreditando unos mejores resultados en las pruebas de condición física realizadas durante la temporada, y a nivel individual se venían implicando en mayor medida en las sesiones de PEVG. La importancia de considerar la interacción de la demarcación con el aspecto a estudiar ya ha sido evidenciada por Del Pino et al. (2015), sobre el perfil de las inteligencias múltiples en función del puesto específico de futbolistas, y en jóvenes futbolistas por Cal ahorro, Torres, Lara \& Zagalaz. (2013).

Respecto a las valoraciones posintervención de los ítems del cuestionario wellness (Tabla 4), encontramos que la percepción de carga de las atacantes es superior a la de las defensas $(4,23$ vs. $3,45, p<0,01)$. Asimismo, las deportistas jóvenes muestran valores superiores que las senior ( 4,12 vs. 3,59, $p<0,001)$, apreciándose resultados similares a los reportados por Francioni et al., 
(2013). Sin embargo, esto no ocurre respecto al ítem esfuerzo, señalando este estudio que son las jóvenes las que declaran menor percepción de esfuerzo. Aunque cabe mencionar que este estudio se realizó con deportistas masculinos y en un contexto de entrenamiento presencial. Por ello, es posible que nuestros resultados pueden estar condicionados por el menor hábito de entrenamiento de las jugadoras jóvenes, y la excepcionalidad del contexto.

Si bien podría haber sido interesante comparar las puntuaciones de la RPE del PEVG con las de los entrenamientos presenciales, no conocemos estudio previo que lo haya realizado a fin de contrastar los resultados hallados en esta experiencia. En este sentido, cabe mencionar que el estudio de Haddad et al. (2013), con 22 futbolistas (17-19 años y al rededor de 6 años de experiencia en alto nivel), concluye que el estrés y la fatiga no alteraban la percepción del esfuerzo en ejercicios submáximos de carrera ( $75 \%$ de la FC máxima de reserva), por lo que creemos que los datos obtenidos en el PEVG no serían diferentes a los reportados en las sesiones presenciales, y que la situación de pandemia vivida no debería modificar las puntuaciones ofrecidas por las deportistas, sino que estas diferencias se deberían a cómo se desarrollaba el proceso de entrenamiento (espacio, tiempo, materiales, etc.), tal y como hallan Blauwet et al. (2020), en la aplicación de programas tradicionales y virtuales de deporte adaptado.

Al comparar los resultados aquí expuestos con programas presenciales similares, se aprecian valores superiores respecto a la RPE que los encontrados por Del Campo (2004) con 10 equipos de baloncesto durante un programa de ocho semanas; y los de Peres et al. (2011); quienes además hallan valores similares entre la RPE prevista por el entrenador de $(3,09)$ y la RPE percibida por los jugadores $(3,17)$, al igual que los resultados de Cuadrado (2010) con 13 jugadores profesionales de balonmano durante un programa de 21 sesiones. Esto nos lleva a pensar que la situación de confinamiento en las que se llevó a cabo este programa ha provocado un aumento en la percepción de carga, aunque creemos que fisiológicamente el trabajo físico realizado ha sido inferior que el que se acostumbraba a hacer de manera presencial. Pero es necesaria mayor investigación al respecto, ya que no se tomaron medidas fisiológicas al finalizar el PEVG para poder comparar con los datos recolectados durante la fase presencial previa, y así poder comprobar si el trbajo virtual produce adaptaciones condicionales similares a las del entrenamiento presencial.
En cuanto a las NPB destacan las al tas puntuaciones halladas en el apoyo a la relación social, lo que puede estar relacionado con el empleo de la aplicación digital que ha permitido un desarrollo sincrónico de los entrenamientos, en los que las jugadoras se veían y se percibían como grupo. Al respecto González et al. (2015), señalan la importancia que tiene el ambiente social para satisfacer las N PB de las jóvenes. Lapercepción de competencia puede relacionarse, en este PEVG, con la rela ción de los valores medios de la percepción de carga $(3,89)$ y el valor medio del nivel de esfuerzo $(4,42)$ relacionado con lamovilización volitiva de recursos para dar respuesta a una tarea. Este aspecto se relaciona con los postulados de Pérez (2017), para quien una alta RPE en las jugadoras se produce por la promoción de un clima hacia el ego en las tareas de potencia æeróbica por parte de los entrenadores.

Asimismo, un ambiente de aprendizaje estructurado y secuenciado, en el que se ofrezca una información comprensible y detallada de los objetivos planteados, fomenta el apoyo a la competencia ( J ang, Reeve $\&$ Deci 2010). Este hecho, se aprecia en la estructura de entrenamientos que se venía realizando tanto en la fase presencial como en la virtual. Del mismo modo, el que la percepción de autonomía hayaal canzado también un valor alto, a pesar de que los entrenamientos estaban prefijados mediante las correspondientes planillas, ha podido estar con el estilo interpersonal de apoyo a la autonomía del preparador físico (Aguirre et al., 2016; Balaguer et al. , 2012; y Cantú-Berrueto et al., 2016), permitiendo a al gunas jugadoras la dirección de determinadas tareas, tal y como se ha visto en diferentes edades y deportes (Adie et al., 2008, 2012; López-Walle et al., 2012). Y promoviendo las iniciativas adoptadas por las jugadoras para implicar a algún miembro de su familia en el desarrollo de los RCO. El apoyo a la NPB de autonomía en el entrenamiento virtual, se puede ver beneficiado con una interacción preparador físico-jugadoras más flexible, que tome más en cuenta la perspectiva de las jugadoras dando opción a la propuesta de ejercicios alternativos, a los inicialmente propuestos por el preparador físico, sobre el aspecto a trabajar en la sesión (estilo interpersonal de apoyo a la autonomía). Este enfoque de promover espacios de autonomía en las sesiones de preparación física, puede reforzar el compromiso de las jugadoras con el mantenimiento y mejora de su condición física, a la vez que se amplían sus conocimientos sobre los contenidos de entrenamiento y el cuidado de su cuerpo, aspecto que les afecta directamente en su desempeño deportivo. 


\section{Conclusiones}

A nivel condicional el empleo de un cuestionario wellness durante el proceso ha permitido proporcionar información al cuerpo técnico acerca del control de la carga de trabajo y la asimilación de las mismas de ma nera individual por cada jugadora. En este sentido, resulta necesaria una mayor investigación respecto a las mejoras físicas desarrolladas y su transferencia a la mejora del rendimiento deportivo en este tipo de PEVG con el objeto de confirmar su eficacia.

La percepción de esfuerzo, tomando al grupo en su conjunto, parece haber estado influenciada por las limitaciones de movilidad impuestas y la duración del esta do de confinamiento, y el PEVG, reflejándose en un aumento secuencial de la percepción de carga del entrenamiento, que alcanzó una mayor puntuación en la fase final. La puntuación máxima del estado de ánimo al canzada en la fase media ha podido ser la consecuencia de un proceso de adaptación generado en la fase inicial, $y$ al efecto de acumulación de fatiga en la fase final.

Durante el proceso del PEVG y atendiendo al perfil de las jugadoras, se aprecian diferencias significativas en función del puesto específico respecto a la percepción de carga, siendo las atacantes las que perciben una ma yor carga en la fase 1, y las sesiones tipo 2 y 3 . En las puntuaciones al finalizar el PEVG, y atendiendo al puesto específico y edad, se encuentran diferencias significativas en la percepción de carga, mostrando unas puntua ciones superiores las jóvenes respecto a las senior, y las atacantes respecto a las defensas. Estos resultados aconsejan considerar los parámetros normativos, puesto específico y edad, en el seguimiento del proceso de entrenamiento.

El tipo de actividades utilizadas y el procedimiento empleado han creado las condiciones necesarias para incrementar la motivación intrínseca a través del apoyo a las NPB, especialmente la de relación social. Lo cual debe ser uno de los aspectos a tener en cuenta a la hora de planificar, organizar y seleccionar las estrategias de trabajo en los PEVG, especialmente en contextos de confinamiento prolongado para mantener la motivación y el nivel de esfuerzo de las deportistas.

\section{Aplicaciones prácticas}

El empleo de una plataforma digital en un modelo de PEVG ofrece una alternativa eficiente para el apoyo a la NPB de relación social, e invita a su consideración en la planificación de este tipo de entrenamiento, espe- cialmente en los deportes colectivos.

LaRPE se muestra como una opción eficiente para el seguimiento y ajuste de la carga en los PEVG, aportando una valoración cuasi-instantánea de la carga percibida y desarrollada durante el entrenamiento, de interés para adecuar las cargas de trabajo de manera individual a las necesidades de cada deportista, lo que ayudará a prevenir lesiones y valorar la planificación frente a la acción, y detectar las situaciones de tensión del entrenamiento.

Entre las limitaciones de este estudio se encuentra el haber medido únicamente al final de la experiencia las NPB; así como no haber considerado el período menstrual de las deportistas como posible influenciador de las conductas (Guijarro, de laVega, \& delValle, 2009; A rias et al., 2018) la calidad del sueño (López-Flores et al. , 2020), el contraste de la cuantificación de la carga con métodos objetivos como dispositivos GPS o simila res y lo reducido de la muestra, hecho que sin duda puede condicionar los resultados aquí expuestos, aunque no menoscaba la importancia de este tipo de estudios sobre PEVG en situaciones singulares.

\section{Referencias}

Adie, J. W., Duda, J. L., \& N toumanis, N. (2008). Autonomy support basic need satisfaction and the optimal functioning of adult male and female sport participants: A test of basic needs theory. M otivation and Emotion, 32, 189-199.

Adie, J. W., Duda, J. L., \& N toumanis, N. (2012). Perceived coach-autonomy support, basic need satisfaction and the well- and ill-being of elite youth soccer players: A longitudinal investigation. Psychology of Sport and Exercise, 13, 51-59.

Aguirre, H. B., Tristán, J. L., López, J. M., Tomás, I., \& Zamarripa, J. I. (2016). Estilos interpersonales del entrenador, frustración de las necesidades psicológicas básicas y el burnout: un análisis longitudinal en futbolistas. Retos. N uevas tendencias en educación física, deporte y recreación, (30), 132-137.

Alexiou, H. \& Coutts, A. J. (2008). A comparison of methods used for quantifying internal training load in women soccer players. International Journal of Sports Physiology and Performance, 3(3), 320-330.

Álvarez, L., López, I., M., Echávarri, J. M., Q uilez, J., Terreros, J. L., \& M anonelles, P. (2009). Análisiscientífico de diferentes métodos de entrenamiento en el fútbol sala. Archivos de medicina del deporte, 26(130), 93-103. 
Álvarez, J. \& Murillo, V. (2016). Comparación entre las cargas planificadas y ejecutadas en el entrenamiento de fútbol sala: la doble escala. Retos: nuevas tendencias en educación física, deporte y recreación, 29(1), 48-52.

Anderson, B. (2017). Estirándose: Guía completa de estiramientos. Barcelona: RBA.

Arias, E. R., Martínez, J. E., Goyes, F. O., Ortiz, V. F., $\&$ M ontero, S. (2018). Variabilidad en el rendimiento físico de las jugadoras de fútbol según las fases del ciclo menstrual. EmásF: revista digital de educación física, (51), 11-3.

Asociación Médica Mundial (2017). Declaración de Helsinki de la AMM - principios éticos para las investigaciones médicas en seres humanos. Recuperado de https:/ / www.wma. net/ es/ policies-post/ declaracion-de-helsinki-de-la-amm-principioseticos-para-las-investigaciones-medicas-en-sereshumanos/

Baena-Extremera, A., Gómez-López, M., GraneroGallegos, A., \& Martínez-M olina, M. (2016). Modelo de predicción de la satisfacción y diversión en Educación Física a partir de la autonomía y el clima motivacional. Universitas Psychologica, 15(2), 39-50.

Balaguer, I. (2010). Clima motivacional y bienestar: Actuación de los entrenadores. En J. M. Buceta \& E. Larumbe (Eds.) Experiencias en psicología del deporte (pp. 181-202). Madrid: Dykinson.

Balaguer, I., González, L., Fabra, P., Castillo, I., Mercé, J., \& Duda, J. L. (2012). Coaches' interpersonal style, basic psychological needs and the well-and ill-being of young soccer players: A longitudinal analysis. Journal of sports sciences, 30(15), 1619-1629.

Blauwet, C. A., Robinson, D., Riley, A., MacEwan, K., Patstone, M., \& Dubon, M. E. (2020). Developing a Virtual Adaptive Sports Program in Response to the COVID 19 Pandemic. PM\&R. https:// doi.org/ 10.1002/ pmrj. 12481

Boyle, M. (2017). El entrenamiento funcional aplicado a los deportes. Madrid:Tutor.

Borg, G. (1998). Borg's Perceived exertion and pain scales. Champaign, IL: Human Kinetics.

Borg, G., \& Dahlstron, H. (1962). A case study of perceived exertion during a work test. Acta Societatis Medicorum U psaliensis, 67, 91-93.

Borresen, J. \& Lambert, M.I. (2008). Q uantifying training load: A comparison of subjective and objective methods. International Journal of Sports Psychology Performance, 3(1), 16-30.

Calahorro, F., Torres, G., Lara, A. J., \& Zagalaz, M. L. (2013). Exigencia competitiva en jugadores de fút- bol cadetes en relación al puesto específico. Ebalonmano. com: Revista de Ciencias del Deporte, 9(1), 2736.

Cantú-Berrueto,A., Castillo, I., López-Walle,J.,Tristán, J., \& Balaguer, I. (2016). Estilo interpersonal del entrenador, necesidades psicológicas básicas y motivación: un estudio en futbolistas universitarios mexicanos. Revista Iberoamericana de Psicología del ejercicio y el deporte, 11(2), 263-270.

Casamichana, D. \& Castellano, J. (2013). Utilidad de la escalade percepción subjetivadel effuerzo paracuantificar la carga de entrenamiento en fútbol. Futbol pf: Revista de Preparación Física en el Fútbol, 8, 53-70.

Castellano, J., Echeazar ra, I., \& Estéfano, I. (2017). Comparación de las demandas físicas en jugadores de fútbol sub13 y sub14 en un 7:7 disputado en diferentes dimensiones. Cultura Ciencia Deporte, 12(34), 55-65.

Cecchini, J. A., Carriedo, A., \& Méndez-Giménez, A. (2019). Testing a circular, feedback model in physical education from self-determination theory. TheJ ournal of Educational Research, 112(4), 473-482.

Coutts, A. J., Rampinini, E., Marcora, S. M., Castagna, C., \& Impellizzeri, F. M. (2009). Heart rate and blood lactate correlates of perceived exertion during small-sided soccer games. Journal of Science and $\mathrm{Me}$ dicine in Sport, 12(1), 79-84. https:/ / doi.org/ 10.1016/ j.jsams. 2007.08.005

Cuadrado, J. (2010). Análisis de la influencia de la intensidad del entre-namiento sobre variables de control de la carga interna en deportes colectivos. Tesis doctoral. Universidad de Granada.

Deci, E. L. \& Ryan, R. M. (2000). The «what» and «why» of Goal Pursuits: of Behavior Human Needs and the Self-determination. Psychological Inquiry, 11(4), 227269. doi:10.1207/ S15327965PLI1104

Del Campo, J. (2004). La intensidad del entrenamiento en jugadores debaloncesto medida a través dela percepción del esfuerzo y la fatiga. Tesis doctoral. Universidad Politécnica de Madrid.

Del Pino, J. M., Gómez, E., \& M oreno, S. (2015). Inteligencias múltiples y rendimiento en fútbol. Apunts. Educación Física y Deportes, 121(3), 44-55.

Duda, J. L. \& Hall, H. K. (2001). Achievement goal theory in sport: Recent extensions and future directions. In R. N. Singer, H. A. Hausenblas, \& C. $M$. Janelle (Eds.), Handbook of research in sport psychology (pp. 417-443). New York:W iley.

Falces, M., Casamichana, D., \& Suárez-Arrones, L. (2013). Comparación de la carga interna en un jue- 
go reducido con presencia/ ausencia del entrenador. Conference: ICTS. II International CongressonTeam Sports.

Francioni, F., Figueiredo, A., Condello, G., Corrado, L., Capranica, L., \& Tessitore, A. (2013). Session$\mathrm{RPE}$ responses to training and competition of two youth soccer teams, diversified by one year of chronological age and participating in the same championship. Annals of Research in Sport and Physical Activity, 4. DOI: 10.14195/2182-7087 411

Gallo, T., Cormack, S., Gabbett, T., W illiams, M., \& Lorenzen, C. (2015). Characteristics impacting on session rating of perceived exertion training load in Australian footballers. Journal of SportsSciences, 33(5), 467-475.

Gaudino, P., laia, F. M., Strudwick, A. J., Hawkins, R. D. , Alberti, G., Atkinson, G., \& G regson, W. (2015). Factorsinfluencing perception of effort (session rating of perceived exertion) during elite soccer training International journal of sports physiology and performance, 10 (7), 860-864. DO I: http:/ / dx.doi.org/ 10.1123/ ijspp.2014-0518

Gonzál ez, L., Castillo, I., García-Merita, M., \& Balaguer, I. (2015). A poyo a la autonomía, satisfacción de las necesidades psicológicas y bienestar: Invarianza de un modelo estructural en futbolistas y bailarines. Revista de Psicología del D eporte, 24(1), 121-129.

Guijarro, E., de laVega, R., \& del Valle, S. (2009). Ciclo menstrual, rendimiento y percepción del esfuerzo en jugadoras de fútbol de élite. Revista Internacional de M edicina y Ciencias de la Actividad Física y el D eporte, 9(34), 96-104.

Haddad, M., Chaouachi, A., Wong, D. P., Castagna, C., Hambli, M., Hue, O., \& Chamari, K. (2013). Influence of fatigue, stress, muscle sorenessand sleep on perceived exertion during submaximal effort. Physiology \& Behavior, 119 (2), 185-189. doi:10.1016/ j. physbeh. 2013.06.016

Hetzler, R. K., Seip, R. L., Boutcher, S. H., Pierce, E., Snead, D., \&Weltman, A. (1991). Effect of exercise modality on ratings of perceived exertion at various lactate concentrations. M edicine and Science in Sports and Exercise, 23(1), 88-92.

Jang, H., Reeve, J., \& Deci, E. L. (2010). Engaging students in learning activities: It is not autonomy support or structure but autonomy support and structure. Journal of Educational Psychology, 102(3), 588.

López-Flores, M., Rodríguez, A., Suárez, D., Rodríguez, J. A., \& Villa, J. G. (2020). Validez de la pulsera de cuantificación Fitbit Flex ${ }^{\circledR}$ en la valoración del sueño. Cultura, Ciencia y D eporte, 15(43), 35-41.

López-Walle, J., Balaguer, I., Castillo. I., \& Tristán, J. (2012).Autonomy support, basic psychological needs and well-being in M exican athletes. TheSpanish Journal of Psychology, 15, 1283-1292.

LosArcos, A., Yanci, J., M endiguchia, J., \& Gorostiaga, E. M. (2014). Rating of muscular and respiratory perceived exertion in professional soccer players. The Journal of Strength \& Conditioning Research, $28(11)$, 3280-3288.

Lovell,T.W., Sirotic,A. C., Impellizzeri, F. M ., \& Coutts, A. J. (2013). Factors affecting perception of effort (session rating of perceived exertion) during rugby league training International journal of sports physiology and performance, $8(1), 62-69$.

Maehr, M. L. \& Zusho, A. (2009). Achievement goal theory: The past, present, and future. In Handbook of motivation at school (pp. 77-104). Retrieved from htt p: / / search. ebscohost.com / login. aspx?direct=true $\alpha d b=p s y h \& A N=2009$ 24219-005\&lang=it\&site= ehost-live

MacM illan, J. H. (2008). Assessment Essentialsfor StandardsBased Education. N ew York. Corwin Press.

Melim, A. M., Petiot, G. H., Clemente, F. M., Nakamura, F.Y., \&Aquino, R. (2020). Hometraining recommendations for soccer players during the COVID-19 pandemic. SportR $\div$ iv. https:/ / doi.org/ 10.31236/ osf.io/ ycwvj

M ontero, I. \& León, O. G. (2007). A guide for naming research studies in Psychology. International Journal of clinical and Health psychology, 7(3), 847-862.

Moras, G. (2017a). El entrenamiento tridimensional y cuadrimensional de la fuerza en los deportes colectivos. En F. Seiru.lo, (2017) El entrenamiento en los deportes de equipo (pp. 82-115). Barcelona: M astercede.

Moras, G. (2017b). La amplitud de movimiento como capacidad facilitadora de la fuerza. En F. Seiru·lo, (2017) El entrenamiento en los deportes de equipo (pp. 167-191). Barcelona: Mastercede.

Moreno-Murcia, J. A., Cervelló, E., Montero, C., Vera, J. A., \& García-Calvo, T. (2012). M etas sociales, necesidades psicológicas básicas y motivación intrínseca como predictores de la percepción del esfuerzo en las clases de educación física. Revista de Psicología del Deporte, 21(2), 215-221.

Moscoso, D. (2020). El contexto del deporte en España durante la crisis sanitaria de la COVID-19. Sociología del deporte, 1(1), 15-19.

Muñoz, J., Castillo, D., \&Yanci, J. (2018). Análisis de la 
percepción subjetiva del esfuerzo diferenciado y de la fatiga en distintos formatos de juegos reducidos en fútbol. Revi sta Iberoamericana de Ciencias de la Actividad Física y el Deporte, 7(2), 14-28.

OMS (2010). Global recommendations on physical activity for health. Geneve: World Health O rganization Library.

Pascual, N., Llorca, V., Carbonell, J. A., \& PerezTurpin, J. A. (2016). A nálisis de la carga interna en los entrenamientos de fútbol sala femenino de la división nacional. Sportis Scientific Technical Journal of School Sport, Physical Education and Psychomotricity, 2(2), 254 267. DOl: http:// dx.doi.org/ 10.17979/ sportis. 2016.2.2.1456

Peres, J., Debora, A., \& Del Campo, J. (2011). Evalua ción de la intensi-dad del entrenamiento de baloncesto a través del salto vertical y RPE. EFdeportes, 152, 1-2. Recuperado de http:// www. efdeportes. com/ efd152/ la-intensidad-delentrenamiento-de-bal oncesto. htm

Pérez, M. (2017). Importancia de las variables psicológicas en la Percepción del Esfuerzo de jóvenes futbolistas. Tesis doctoral no publicada Universidad de Valencia, Va lencia.

Pescatello, L. S., Arena, R., Riebe, D., \& Thompson, P. D. (2014). Guidelines for exercise testing and prescription (9 ed. ). Philadelphia: Wolters K luwer/ Lippincott W illiams \& W ilkins Health.

R Core Team (2017). R: A language and environment for statistical computing. R Foundation for Statistical Computing, Vienna, Austria. Recuperado de https:/ / www. R-project.org/ .

Real Decreto 463/ 2020, de 14 de marzo, por el que se declara el estado de alarma para la gestion de la situacion de crisis sanitaria ocasionada por el COVID19. Boletín 0 ficial del Estado, 14 de marzo de 2020, núm. 67, pp. 25390 a 25400.

Rebelo, A. N. C., Silva, P., Rago, V., Barreira, D., \& K rustrup, P. (2016). Differencesin strength and speed demands between $4 \mathrm{v} 4$ and $8 \mathrm{v} 8$ small-sided football games. Journal of sports sciences, 34(24), 2246-2254.

Ryan, R. M. \& Deci, E. L. (2000). Self-determination theory and the facilitation of intrinsic motivation, social development, and well-being. American Psychologist, 55(1), 68- 78.

Ryan, R. M., \& Deci, E. L. (2019). Brick by brick:The origins, development, and future of selfdetermination theory. In Advances in M otivation Science, 6, 111-156.

Robbins, S. (2004). Comportamiento Organizacional. 10a
Edición. México: Pearson Educación.

Salazar-Ayala, C. M. \& Gastélum-Cuadras G. (2020).

Teoría de la autodeterminación en el contexto de educación física: Unarevisión sistemática. Retos: nuevas tendencias en educación física, deporte y recreación, (38), 838-444.

Seguí-U rbaneja, J., Julião, R. P., N ogueira, R. M., Dorado, V., \& Farías, E. I. (2021). Impacto delaCOVID19 en la práctica deportiva de personas participantes en eventos deportivos de carrera a pie y ciclismo en España y Portugal. Retos: nuevas tendencias en educación física, deportey recreación, 39(1), 743-749

Sevil, J., Julián, J., A barca-Sos, A., Aibar, A., \& GarcíaGonzález, L. (2014). Efecto de una intervención docente para la mejora de variables motivacionales situacionales en Educación Física. Retos. Nuevas Tendenciasen Educación Física, D eportey Recreación, 26, 108113.

Sevil, J., Abós, Á., Generelo, E., Aibar, A., \& GarcíaGonzález, L. (2016). Importancia del apoyo a las necesidades psicológicas básicas en la predisposición hacia diferentes contenidos en Educación Física. Retos. N uevastendencias en educación física, deportey recreación, 29, 3-8.

Siedentop, D. (1998). Aprender a enseñar la educación física. Barcelona: Inde.

Tenenbaum, G., Hall, H. K., Calcagnini, N., Lange, R., Freeman, G., \& Lloyd, M. (2001). Coping with physical exertion and negative feedback under competitive and self-standard conditions. Journal of Applied Social Psychology, 31(8), 1582-1626.

Viru, A., \& Viru, M. (2003). Análisis y control del rendimiento deportivo. Barcelona: Paidotribo

Vlachopoulos, S. P. \& Michailidou, S. (2006). Development and initial validation of a measure of autonomy, competence, and relatedness in exercise: The Basic Psychological Needs in Exercise Scale. Measurement in physical education and exercise science, 10(3), 179-201.

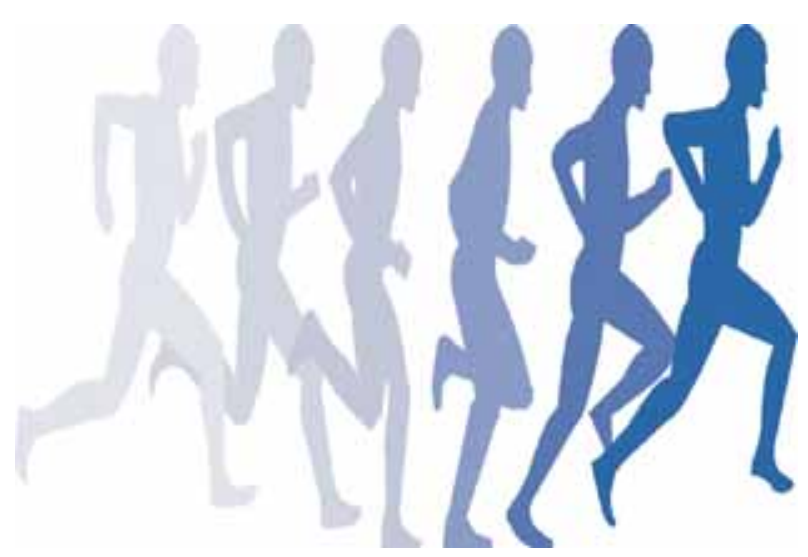

